ESAIM: PROCEEDINGS, August 2009, Vol. 28, p. 1-12

M. Ismail, B. Maury \& J.-F. Gerbeau, Editors

\title{
MATHEMATICAL MODELLING OF THE ATHEROSCLEROTIC PLAQUE FORMATION ${ }^{*}, * *$
}

\author{
Vincent Calvez ${ }^{1}$, Abderrhaman Ebde ${ }^{2}$, Nicolas Meunier ${ }^{3}$ and Annie Raoult ${ }^{3}$
}

\begin{abstract}
This article is devoted to the construction of a mathematical model describing the early formation of atherosclerotic lesions. Following the work of El Khatib, Genieys and Volpert [2], we model atherosclerosis as an inflammatory disease. We consider that the inflammatory process starts with the penetration of Low Density Lipoproteins cholesterol in the intima. This phenomenon is related to the local blood flow dynamics. Using a system of reaction-diffusion equations, we first provide a one-dimensional model of lesion growth. Then we perform numerical simulations on a two-dimensional geometry mimicking the carotid artery. We couple the previous mathematical model with blood flow and we provide a model in which the lesion appears in the area of lower shear stress.
\end{abstract}

Résumé. Cet article est consacré à la construction d'un modèle mathématique décrivant la formation de lésions précoces dans l'athérosclérose. Suite au travail de El Khatib, Genieys and Volpert [2], nous modélisons l'athérosclérose comme une maladie inflammatoire. Nous considérons que le procesus inflammatoire débute avec la pénétration de "Low Density Lipoproteins cholesterol" dans l'intima. Ce phénomène est relié à la dynamique locale de l'écoulement du sang. En utilisant un système d'équations de réaction-diffusion, nous donnons tout d'abord un modèle monodimensionel de croissance de lésion. Puis, dans le cas d'une bifurcation bidimensionnelle, nous couplons le modèle mathématique précédent avec l'écoulement du sang et nous fournissons un modèle dans lequel la lésion apparaît dans la zone de plus faible cisaillement.

\section{INTRODUCTION}

This work is motivated by the design of mathematical models and numerical tools to simulate the first steps in the atherosclerotic plaque formation. The main goal is first to build a mathematical model of the inflammatory process which takes place in the intima (biomolecular process) and then to add to this model the action of the wall shear stress due to the blood flow in the artery (fluid dynamics). Up to our knowledge, this coupling has not been studied yet.

\footnotetext{
* The authors wish to thank Professor Bertrand Maury for having supervised this work.

** This work has been supported by the ANR project MOSICOB and by the Marie Curie Actions of the European Commission in the frame of the DEASE project (MEST-CT-2005-021122).

${ }^{1}$ Unité de mathématiques pures et appliquées ENS Lyon, CNRS UMR 5669; e-mail: vincent.calvez@umpa.ens-lyon.fr

2 Département de mathématiques et applications DMA Ecole Normale Supérieure 45, rue d'Ulm Paris 75230 Cedex 05 France; e-mail: Abderrahman.Ebde@ens.fr

${ }^{3}$ MAP5, Université Paris Descartes 45 rue des Saints Pères 75270 Paris Cedex 06. France;

e-mail: nicolas.meunier@math-info.univ-paris5.fr; Annie.Raoult@parisdescartes.fr

(C) EDP Sciences, SMAI 2009
} 

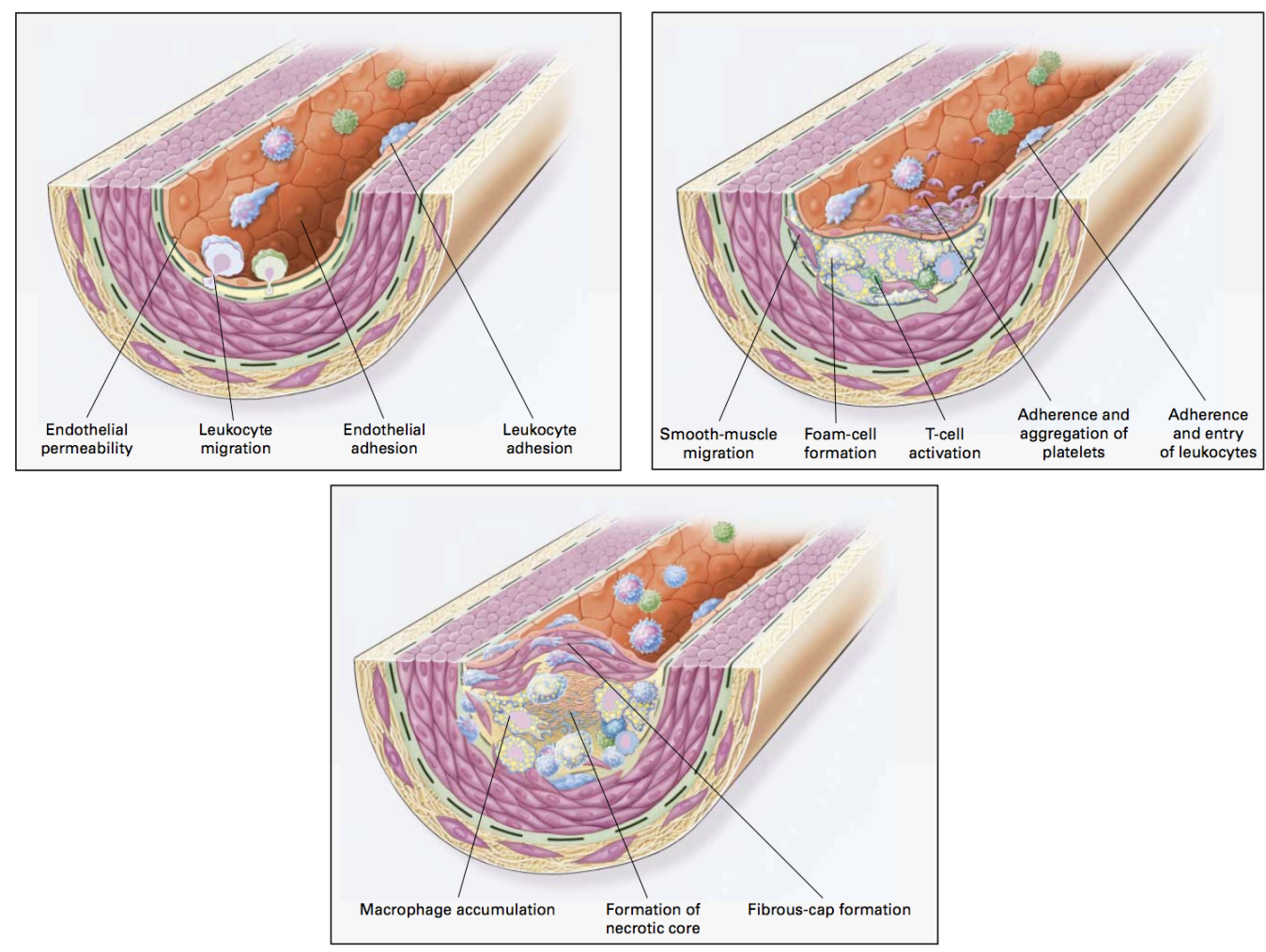

FigURE 1. Early events of atherosclerosis. LDL penetrates in the intima, thus triggering an inflammatory process. The atheromatous plaque is eventually covered by a fibrous cap made of smooth muscle cells, (from R. Ross, The New England Journal of Medicine (1999), [6]).

Atherosclerosis and its complications are one of the most common causes of death in western societies and in Japan. According to recent works (see [6] and the references therein), the lesions of atherosclerosis which take place in the intimal layer constitute a chronic inflammatory response to injury. It is now well accepted that it is the result of interactions between plasma molecules such as lipoproteins, cellular components and the extracellular matrix of the arterial wall. More precisely, atherosclerosis involves plasma low density proteins cholesterol, monocytes/macrophages, endothelial cells and smooth muscle cells.

The advanced lesions of atherosclerosis are most commonly reported in large or medium arteries such as the abdominal aorta, the coronary arteries or the carotid bifurcation. These arteries can be considered as thickwalled tubes whose internal surface is exposed to flowing blood and external surface is bounded by tissue. The arterial wall contains three distinctive layers which are (from the internal side to the external side) the intima (which is composed of endothelial cells), the media and the adventia, see Figure 1.

The first step in the atherosclerosis process seems to be the accumulation of LDL cholesterol in the intima, where part of it is oxydized and becomes pathological. In order to remove it, circulating immune cells (e.g. monocytes) are recruited. Once in the intima, the monocytes differentiate to become macrophages that ingest by phagocytosis the oxydized LDL. The ingestion of large amounts of oxydized LDL transforms the fatty macrophages into foam cells, see Figure 2. Foam cells are responsible for the growth of a subendothelial plaque which eventually emerges in the artery lumen and are expected to reinforce the inflammatory response as they appear as undesirable bodies. 


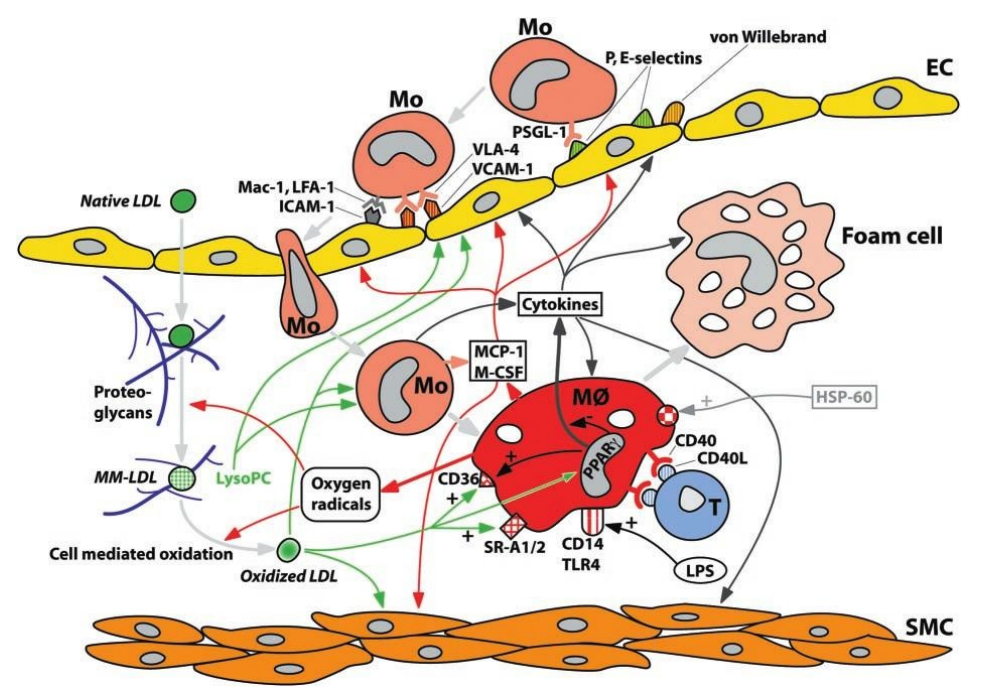

Figure 2. B. Osterud and E. Bjorklid, Role of monocytes in atherogenesis (2003), [5]

Moreover, it appears also that an increase of macrophage concentration in the intima leads to the production of pro-inflammatory cytokines, see [6]. These cytokines contribute to recruit more macrophages.

It appears that local hemodynamics conditions have significant impact on the earliest stages of atherosclerosis lesions, see [7]. Indeed, low wall shear stress, oscillating shear stress and long residence times have been shown experimentally to influence the formation of plaques.

In this work, using reaction-diffusion equations, we provide a mathematical model describing in a simplified manner the inflammatory process, resulting in foam cells accumulation and lesion growth. We ensure the growth of the initial domain by an incompressibility assumption together with the assumption of constant profiles along the normal direction to the interface between the arterial lumen and the wall. The lesion growth equation is then developed in a one-dimensional setting (along the normal direction to the interface). A more general mathematical model of lesion growth will be developed in a further work [1].

Then we consider a 2D bifurcation geometry which is filled by blood, considered as a Newtonian, incompressible fluid governed by Navier-Stokes equations. We numerically compute the shear stress near the boundary and we couple the inflammatory mathematical model with hemodynamic conditions. According to literature, we set in our approach that areas subject to low shear stress are more likely to exhibit plaque emergence.

The major improvement in our two dimensional model of arterial inflammation is that it is set on a domain which evolves with time at a very low velocity compared to the blood velocity. According to our model the inflammation process modifies the shape of the domain due to the infiltration of circulating material. The two time scales are very different: therefore the domain is assumed quasi-staionary for computing the fluid dynamics. More precisely, the first step in our approach is to compute the wall shear stress for a given arterial $2 \mathrm{D}$ geometry on the interface between the arterial lumen and the intima. Then, we solve (numerically) the system of reaction-diffusion equations for the given intima's geometry. We obtain a new value of the height of the intima. This induces a change of the intima's geometry and hence a change of the arterial lumen. For this new geometry, we again compute the wall shear stress on the boundary and so on.

The paper is organized as follows. In the second section, we give the system of partial differential equations that model the inflammatory process. We also provide a lesion growth equation under matter incompressibility assumption in the intima and we give numerical simulations of lesion growth. In the third section we couple the inflammatory model with a blood flow satisfying Navier-Stokes equations in a two dimensional domain. 


\section{Mathematical modelling of ATHERosclerotic Plaque initiation}

We first focus on the inflammatory process and then on its links with hemodynamics.

We summarize and simplify the disease progression described in the previous section by the following steps:

(1) Passive penetration of LDL in the intima. The intima LDL concentration depends on the plasma LDL concentration, which in turn depends on local hemodynamics. In addition, we assume that areas with lower wall shear stress correspond to higher penetration of LDL.

(2) The passive infiltration is immediately followed by an oxydation (i.e. we consider only the fraction of LDL intimal concentration which is oxydized). This is true at least for a fraction of the LDL intimal concentration.

(3) When the oxydized LDL concentration exceeds a threshold, it can be sensed by the endothelial cells. This triggers recruitment of monocytes through the vessel wall.

(4) The incoming monocytes instantaneously differentiate into active macrophages.

(5) Active macrophages absorb oxydized LDL in the intima through a mass action law. This reaction transforms macrophages into foam cells and yields the secretion of a pro-inflammatory signal which contribute to recruit new monocytes.

(6) Newly formed foam cells are responsible for the local volume increase. Under a local incompressibilty assumption, when foam cells are created the intima volume is locally increasing.

Let us now describe the cellular and the chemical species that are to be considered in this model. We restrict ourselves to the following species in the intima: LDL cholesterol, oxydized LDL, immune cells (monocytes/macrophages), foam cells, cytokynes and extracellular matrix (which comprises the smooth cells).

We denote by $C, O x, M, F, S$ and $w$ the concentrations (or densities) of respectively LDL cholesterol, oxydized LDL, macrophages, foam cells, signal (a generic chemoattractant which gathers the cytokynes) and biomass (which includes the extracellular matrix). The previous concentrations are functions of the space variables $x, y$ and of the time $t$. The direction of the blood flow is $x$, and $y$ is the transversal direction through the intima.

We also introduce the transversal averaged value, by integrating the quantity of a cellular species (the LDL cholesterol e.g.). In the case of LDL cholesterol, it is denoted by $Q_{C}$ and it is defined by $Q_{C}=\int_{0}^{h} C \mathrm{~d} y$. We will denote by $Q_{O x}, Q_{M}, Q_{F}$ and $Q_{w}$ this quantity for the other cellular components.

Before giving the equations governing the evolution of the uplisted species, let us comment on the geometrical frame.

\subsection{Geometrical assumption for the intima}

We simply describe the intima as a $2 \mathrm{D}$ band of height $h(x, t)$ with $x \in[0, L]$ and $t>0$. In the sequel, the coordinate $y$, which varies between 0 and $h(x, t)$, will denote the height in the intima.

The boundary $y=h$ corresponds to the interface with the blood while $y=0$ corresponds to the interface with the media. Moreover, we assume that the lower boundary of the intima (i.e. the one which is in contact with the media) is fixed.

Remark 2.1. In future work, we aim at considering the lower boundary of the interface with the media where smooth muscle cells act as an elastic material. We neglect at this time the remodelling effect of the subendothelial tissues in response of a local volume increase. In fact we assume that this remodelling effect is nearly saturated, leading to a push of the endothelial wall.

Remark 2.2. Before the passive infiltration and the inflammatory process, the intima is very thin and it can be considered as a one dimensional domain. In order to keep this property with the finite element solver FreeFem++ which is well suited for two dimensional domains, we shall artificially enlarge the intima and we shall penalize the normal direction (to the interface) by considering a very fast diffusion in that direction. 


\subsection{Local matter incompressibility assumption}

Moreover, we assume that macrophage size (before ingesting oxydized LDL) and oxydized LDL size are small in comparison with size of foam cells (such an assumption could be improved in a further work), so that only the foam cells contribute to the intimal volume.

The local matter incompressibility assumption can be stated as:

There exists $A$ such that $w(x, y, t)+F(x, y, t)=A$, for all $x \in] 0, L[$, for all $y \in] 0, h[$ and for all $t>0$.

We introduce the vector field $v(x, y, t)$ which stands for the rise of the atheromatous plaque from the intima. Furthermore, we assume that the matter growth induced by foam cells accumulation is directed in the $y$ direction. In other words, denoting by $v$ the growth speed, we assume that

$$
\left.\partial_{x} v=0 \text {, for all } x \in\right] 0, L[\text {, for all } y \in] 0, h[\text { and for all } t>0 .
$$

\subsection{Oxydized LDL}

Consider now the partial differential equation modelling the evolution of oxydized LDL concentration:

$$
\left\{\begin{array}{lr}
\partial_{t} O x+\operatorname{div}(v O x)=d_{1} \triangle O x-k_{1} O x \cdot M, & \text { for all }(x, y) \in] 0, L[\times] 0, h[, \\
\partial_{y} O x=\tau(\mathrm{x}) C, & \text { if } y=h, \text { for all } x \in] 0, L[, \\
\partial_{y} O x=0, & \text { if } y=0, \text { for all } x \in] 0, L[.
\end{array}\right.
$$

This equation reflects the conversion of macrophages into foam cells when they ingest oxydized LDL molecules. The law of mass action is questionable, but we choose it in a first step. We use the product of both concentrations since the reaction takes place when oxydized LDL and macrophages are both available in the intima. The second left-hand side term models the lesion growth. It states that oxydized LDL are transported with the tissue displacement having speed $v$.

Remark 2.3. We refer to the recent work of El Khatib, Génieys, Kazmierczak and Volpert [3] where the authors study the existence of $2 \mathrm{D}$ traveling waves in the same context, when the reaction term is supported on the domain's boundary.

Moreover in order to allow a Brownian displacement of oxydized LDL in the intima, we introduce a diffusion term. As the intima is a porous or a solid phase, this diffusion term remains small.

The factor $\tau(x)$ denotes the permeability of the blood vessel. We will come back to that later. The term involving the coefficient $\tau(x)$ is a local reaction term. Its dependance with respect to the variable $x$ constitutes the novelty of this work.

The permeability $\tau(x)$ reflects the biomechanical properties of the endothelial wall which separates the intima from the lumen where blood flows. We discuss in a next section the possible choices for this function. To sum up, we focus on the wall shear stress which is known to favour the penetration of both LDL and macrophages when it is low.

In this section, we consider that the function $\tau$ is a cut-off function of the wall shear stress allowing the activation of the inflammatory reaction in a localized area of the intima where the shear stress is below some threshold. The exact expression of the function $\tau$ will be explained below. 


\subsection{Macrophage}

Let us next explain the partial differential equation which models the evolution of macrophage density:

$$
\left\{\begin{array}{lr}
\partial_{t} M+\operatorname{div}(v M)=d_{2} \triangle M-k_{1} O x \cdot M, & \text { for all }(x, y) \in] 0, L[\times] 0, h[, \\
\partial_{y} M=f(S), & \text { if } y=h, \text { for all } x \in] 0, L[.
\end{array}\right.
$$

In this equation, we assume that the macrophage recruitment depends on a general pro-inflammatory signal $S$ which gathers both chemokines and cytokines. This signal acts through the contribution $f(S)$ which acts on the boundary. This reflects the recruitment of circulating immune cells via adhesion on the endothelial boundary and invagination. The response function $f(S)$ shall be increasing with a saturation effect due to the limited transfer rate of monocytes accross the vessel wall.

In order to impose a limit in this macrophage recruitment we classically choose a function $f$ of the form:

$$
f(S)=\frac{S}{1+S}
$$

We introduce a diffusion term. The diffusion process for macrophages is limited with a diffusion constant even smaller than for the LDL.

Remark 2.4. We will consider in a further work a population of immune cells which is structured by maturity (i.e. the level of absorbed LDL): this framework will enable to better take into account the evolution of macrophages into foam cells and eventually to derive parameters having a clear biological background.

\subsection{Foam cells}

Let us now consider the partial differential equation satisfied by the foam cell concentration:

$$
\left.\partial_{t} F+\operatorname{div}(v F)=k_{1} O x \cdot M \text { for all }(x, y) \in\right] 0, L[\times] 0, h[.
$$

The $k_{1}$ term is a creation term coming from the reaction of absorption of oxydized LDL by macrphages. The second left-hand side term models the lesion growth. It states that foam cells are transported with the tissue displacement having speed $v$. The unknown speed will be derived by the local incompressibility assumption.

Furthermore, contrary to the other cellular species, we assume that the foam cells are inert.

\subsection{Signal}

Consider now the partial differential equation satisfied by the chemoattractant which is a chemical species:

$$
\left\{\begin{array}{lr}
\partial_{t} S=d_{3} \triangle S-\lambda S+k_{1} O x \cdot M, & \text { for all }(x, y) \in] 0, L[\times] 0, h[, \\
\partial_{y} S=\gamma\left(O x-O x^{t h}\right), & \text { if } y=h, \text { for all } x \in] 0, L[, \\
\partial_{y} S=0, & \text { if } y=0, \text { for all } x \in] 0, L[.
\end{array}\right.
$$

The signal can diffuse $\left(d_{3}\right.$ term). Moreover the $\lambda$ term is a degradate rate. Both consumption and production appear in this equation for the chemoattractant. It is created when reaction $M+O x \rightarrow F$ happens and when oxydized LDL concentration exceeds a threshold to initiate the inflammation response.

Finally, the $\gamma$ term is an activation term and $O x^{\text {th }}$ corresponds to a given oxydized LDL quantity which is necessary in order to activate the process. Indeed, the starting point of the signal emission is assumed to be a too high oxydized LDL concentration. 
Remark 2.5. We could have considered as well that the signal is created when the macrophage density is decreasing or when the oxydized LDL concentration increases.

\subsection{Biomass}

We denote by $w$ the biomass which constitutes the rest of the intimal medium (extracellular matrix, smooth cells and other), which do not contribute to the inflammatory process within our assumptions. It satisfies:

$$
\partial_{t} w+\operatorname{div}(w v)=0
$$

\subsection{Lesion speed}

In order to compute the growth speed of the lesion, we add equations (4) and (6) and we use the local incompressibility assumption (1):

$$
\begin{aligned}
\partial_{t}(F+w) & =k_{1} O x \cdot M-\operatorname{div}((F+w) v) \\
0 & =k_{1} O x \cdot M-A \operatorname{div}(v)=k_{1} O x \cdot M-A \partial_{y}\left(v_{y}\right)
\end{aligned}
$$

thanks to the assumption that $v_{x}=0$.

Recalling remark 2.2, we assume that all the concentrations are constant in the $y$-direction allowing to replace the product of average concentration (in the $y$-direction) by the average of the product.

Hence integrating (8) over $y$, we deduce that

$$
\begin{aligned}
v(h)-v(0) & =\frac{k_{1}}{A} \int_{0}^{h} O x \cdot M \mathrm{~d} y \\
& =\frac{k_{1}}{A h} Q_{O x} \cdot Q_{M}
\end{aligned}
$$

We assume that $v(0)=0$ (no intimal remodelling from below). Observe that

$$
v(h)=\frac{\mathrm{d} h}{\mathrm{~d} t},
$$

we deduce that $h$ satisfies the following ordinary differential equation:

$$
\frac{\mathrm{d} h}{\mathrm{~d} t}=\frac{k_{1}}{A h} Q_{O x} \cdot Q_{M} .
$$

To summarize this simple equation in words, we assume that the intima grow vertically due to the creation of foam cells uniformly in height.

Remark 2.6. In our one-dimensional lesion growth model of the intima, we consider that the lesion velocity $v(h(x))$ is the result of the reactions located at at a section $0 \leq y \leq h(x)$. In order to build a two dimensional model we should add some caracteristic law of the atheromatous plaque and this is an open problem.

\subsection{Numerical simulations of the inflammatory process}

The finite element solver FreeFem ++ has been used to run simulations. The result is given in Figure 3: on the left, the intima at rest, on the right, there is the intima after an inflammatory process described above. In 

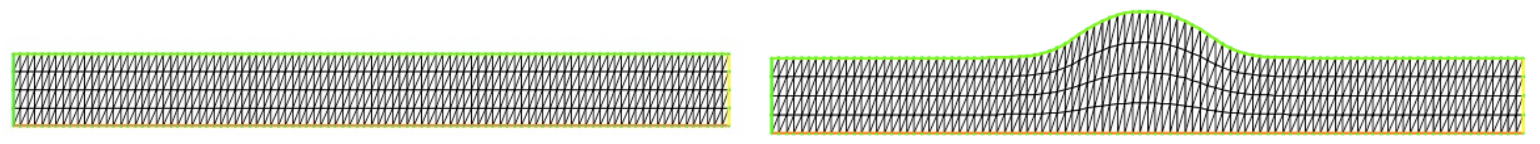

FiguRE 3. Schematic geometry of the intima layer. Top boundary is the interface with the aterial lumen which might be deformed due to the displacement $v$. On the left the intima at rest and the intima after inflammatory reaction on the right

this section, we assume that we can neglect the effect of the transport due to the lesion growth on the oxydized LDL and on the macrophage. The reaction-diffusion equations that are numerically solved are the following:

$$
\left\{\begin{aligned}
\partial_{t} Q_{O x} & =d_{1} \partial_{x x} Q_{O x}-k_{1} Q_{O x} \cdot Q_{M}+\tau(x) C \\
\partial_{t} Q_{M} & =d_{2} \partial_{x x} Q_{M}-k_{1} Q_{O x} \cdot Q_{M}+f\left(Q_{S}\right) \\
\partial_{t} Q_{F}+\partial_{x}\left(Q_{F} v\right) & =k_{1} Q_{O x} \cdot Q_{M} \\
\partial_{t} Q_{S} & =d_{3} \partial_{x x} Q_{S}-\lambda Q_{S}+k_{1} Q_{O x} \cdot Q_{M}+\gamma\left(Q_{O x}-Q_{O x^{t h}}\right) .
\end{aligned}\right.
$$

Furthermore, the height $h(x, t)$ satisfies the O.D.E.:

$$
\frac{\mathrm{d} h}{\mathrm{~d} t}=\frac{k_{1}}{A h} Q_{O x} \cdot Q_{M}
$$

In this section we take

$$
\tau(x)=10^{-3}+\chi_{x^{2}<\sigma^{2}}(x),
$$

where $\chi_{A}(x)$ is the characteristic function associated with the set $A$, where $\sigma=0.4$.

According to [4] the physical and biological parameters considered for the numerical simulations are the following:

$$
\begin{aligned}
& d_{1}=\quad d_{3}=10^{2} \times d_{2}=10^{-3} \mathrm{~cm}^{2} \cdot \mathrm{s}^{-1} \\
& C_{\mathrm{LDL} \text { in }}^{\text {initial }} \text { the intima }= \\
& Q_{\mathrm{LDL} \text { in the intima }}^{\text {initial }}= \\
& Q_{O x}^{t h}= \\
& 0.1 \mathrm{~g} \cdot \mathrm{cm}^{-3} \\
& A= \\
& 10^{-3} \mathrm{~g} \cdot \mathrm{cm}^{-1} \\
& 0.25 \mathrm{~g} \cdot \mathrm{cm}^{-1} \\
& t= \\
& 1 \mathrm{~g} \cdot \mathrm{cm}^{-3} \\
& \lambda= \\
& \text { caracteristic time of the lesion growth }=10^{7} \mathrm{~s} \\
& h_{0}= \\
& 10 s^{-1} \\
& k_{1}= \\
& \text { intima intitial height }=10^{-2} \mathrm{~cm} \\
& \gamma= \\
& 1 \mathrm{~cm} \cdot \mathrm{g}^{-1} \cdot \mathrm{s}^{-1} \\
& 1 s^{-1} \text {. }
\end{aligned}
$$

Moreover the initial conditions for $Q_{O x}, Q_{M}, Q_{F}$ and $Q_{S}$ are:

$$
Q_{O x}^{\text {initial }}=0, Q_{M}^{\text {initial }}=0.1, Q_{F}^{\text {initial }}=0, Q_{S}^{\text {initial }}=0 .
$$




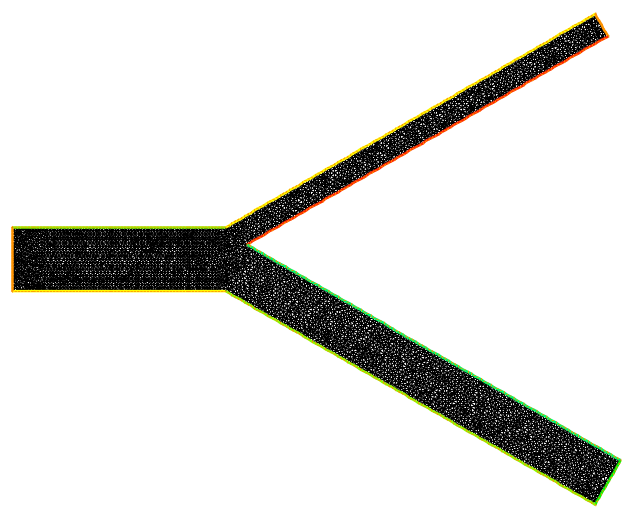

Figure 4. Two dimensional bifurcation geometry $\mathcal{A}(0)$ modelling the artery

\section{BLOOD FLOW IN THE ARTERY}

In this section, we describe the blood flow in the artery. We suppose that the artery is filled with a Newtonian, incompressible fluid. We denote by $\mathcal{A}(t)$ the domain representing the artery at time $t>0$, where the fluid velocity is set to zero. We assume that the domain $\mathcal{A}(t)$ is not fixed. Indeed, the arterial wall, which separates the intima from the artery is evolving.

In the artery $\mathcal{A}(t)$, we assume that the fluid obeys Navier-Stokes equations:

$$
\left\{\begin{aligned}
\left(\frac{\partial u}{\partial t}+u \cdot \nabla u\right)-\nu \Delta u+\nabla p= & f \\
\operatorname{div} u= & 0 .
\end{aligned}\right.
$$

Here $u$ stands for the velocity field, $p$ for the pressure, $\nu$ for the dynamic viscosity. Furthermore, we impose a no-slip condition: at each point of the lateral boundary of $\mathcal{A}(t)$ the velocity of the fluid is equal to zero.

For all time step $t>0$, we compute the wall shear stress on the lateral boundary $\Sigma(t)$ of $\mathcal{A}(t)$ (recall that the lower boundary $\Sigma(t)$ of $\mathcal{A}(t)$ is the curve $x \rightarrow h(x, t))$. To do so, we use the classical form of wall normal shear stress $t \cdot \sigma \cdot n$, where $\sigma$ is the Cauchy shear stress tensor and $t, n$ are the tangential vector, inwards vector (in the artery) normal to the boundary, with

$$
\sigma=\mu\left(\nabla u+\nabla u^{t}\right)-p \text { Id. }
$$

Remark 3.1. It is to be noticed that our approach is very different from the approach used to deal with problems of fluid/structure interaction, because of the separation between the timescale of the fluid and the timescale of the lesion growth. Indeed, in our approach the blood flow does not directly affect the geometry of the artery. The geometry changes if the intima's height increases. The reason of such an increase is the inflammatory process. As we shall see below, this inflammatory process is related with area on the lower boundary $\Sigma(t)$ where the wall shear stress is the lowest. This is the $\tau$ boundary term in (2).

Let us now describe the geometry of the artery. At time $t=0, \mathcal{A}(0)$ is given by a two-dimensional bifurcation geometry, see Figure 4. At time $t=0$, the interface between the arterial lumen and the intima is defined by 


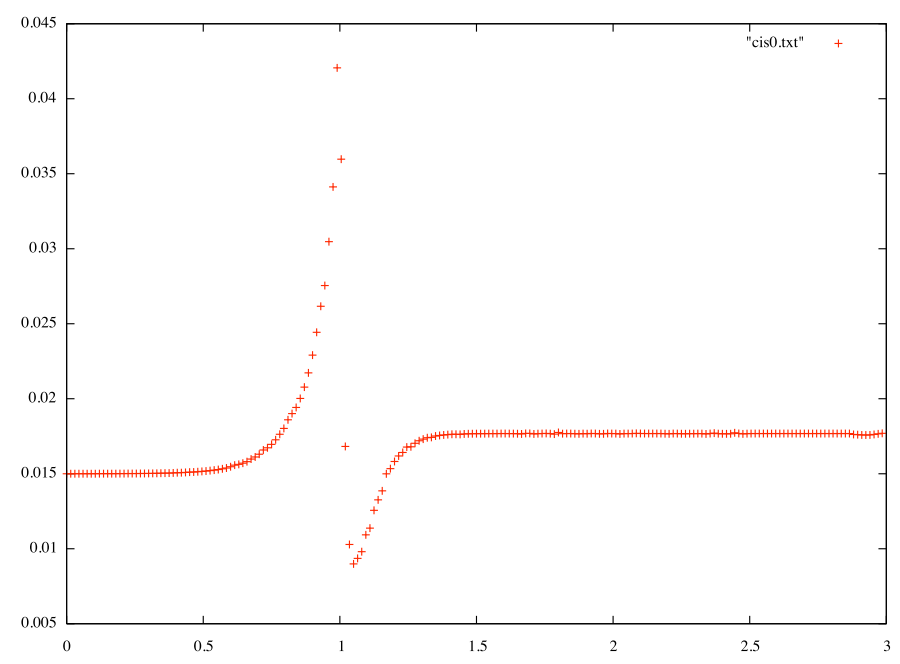

FiguRE 5. Wall shear stress on the lower boundary $\Sigma(0)$ of the two dimensional geometry $\mathcal{A}(0)$ represented in Figure 4.

the following function:

$$
\left\{\begin{array}{l}
0 \leq t \leq 1, x=t ; y=0 \\
0 \leq t \leq 2, x=1+t \cos (\pi / 6) ; y=-t \sin (\pi / 6)
\end{array}\right.
$$

At the inlet, we impose a symmetric Poiseuille speed profile. We use FreeFem++ to compute the velocity in this geometry. Then we compute the wall shear stress on the lower lateral boundary $\Sigma(t)$ of the arterial geometry. We represent this wall shear stress on Figure 5.

\section{Coupling With Blood Flow in the ARTery}

In this section we explain how we couple the inflammatory model with the blood flow in the artery. Let $\Omega=\mathcal{A}(t) \cup \mathcal{I}(t) \subset \mathbb{R}^{2}$ where $\mathcal{A}(t)$ has already been explained and $\mathcal{I}(t)$ represents the intima. For every time $t>0$, first, as explained in the previous section, we numerically compute the blood flow in $\mathcal{A}(t)$ and the wall shear stress denoted by wss on $\Sigma(t)$. Then, we compute the absorption rate $\tau$ as a function of the wall shear stress as follows $\tau(x)=H(w s s(x)), x \in \Sigma(t)$, where $H$ is a Hill function, see (13) below.

Remark 4.1. This assumption is widely accepted in the literature. However we aim at describing in a further work more precisely the advection-diffusion dynamics of the circulating LDL. We hope that this will shed a new light on the LDL being available at the interface with the intima. This description will be strongly motivated by more realistic situations such as oscillating shear stress. Furthermore, it would be also interesting to consider the situation of oscillating blood flow.

Once, we have computed the wall shear stress wss near the lower boundary $\Sigma(t)$, we can obtain (for this given Hill function $H)$ the values of $\tau(x)=H(w s s(x))$ for all $x \in \Sigma(t)$. Then, we numerically solve the following system of reaction/diffusion equations given by (9). The value of $h(x, t)$ is then obtained by solving the O.D.E. (8).

This gives "new geometries" $\mathcal{A}(t)$ and $\mathcal{I}(t)$. For the new geometry $\mathcal{A}(t)$, we numerically compute the blood flow and wall shear stress and this gives a new value of $h$. 


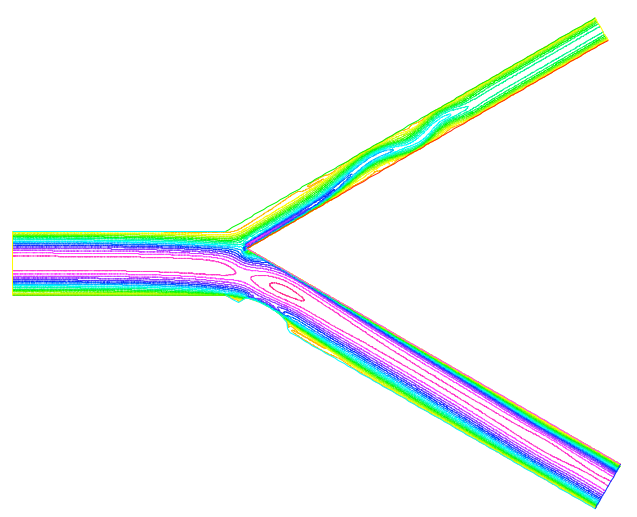

Figure 6. Arterial geometry after 40 iterations: $\mathcal{A}(40)$ and velocity of the fluid.

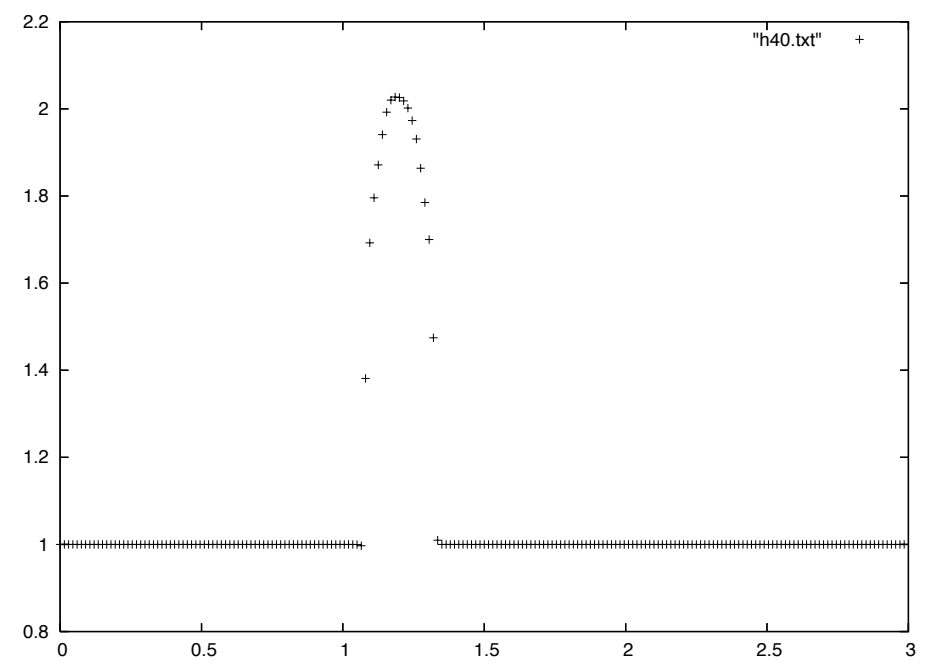

FiguRE 7 . Height corresponding to the intima's geometry after 40 iterations: $h(z, 40)$.

\subsection{Numerical simulations}

We use again the finite element solver FreeFem ++ to run simulations in a two dimensional bifurcation geometry and we obtain the results represented on Figures 7 and 6.

The mesh of the bifurcation geometry modelling the artery contains around 6700 triangles while the mesh modelling the intima (located at the lower side of the artery) contains around 2200 triangles.

Our first step is to run simulations on the undeformed arterial geometry. The fluid circulating is newtonian with $2.510^{-4}$ viscosity and unit density. The boundary condition applied on the inlet is of Dirichlet type 
and it is of natural type on the outlets. A first simulation (during 10 iterations) has been performed with FreeFem ++ in this undeformed geometry for a Stokes flow. Then during 20 iterations a second simulation has been performed with FreeFem++ in this undeformed geometry for a Navier-Stokes flow.

After this 30 iterations, we compute the wall shear stress and run simulations with the inflammatory model in the intima with a function $\tau$ which is one in area with lower wall shear stress. We perform simulations with the reaction-diffusion equations during a few iterations.

The inflammatory model with lesion growth induces a new geometry after these iterations. We then run simulations on the deformed arterial geometry.

After these fluid iterations we update the intimal height and so on.

During the numerical simulations we chose for the Hill function the function given by:

$$
\tau(x)=H(w s s(x))=\sqrt{\frac{(w s s-(1+\sigma))_{+}(1+3 \sigma-w s s)_{+}}{\sigma^{2}}}
$$

where (.) + denotes the positive part, $0 \leq x \leq 3$ is associated with the parametrization $(12)$, wss $(x)$ is the wall shear stress taken at $x$ and $\sigma=0.1$ has been chosen such that the region where $(w s s-(1+\sigma))_{+}(1+3 \sigma-w s s)_{+}>0$ corresponds to region where the wall shear stress is the lowest for the interface between the arterial lumen and the intima.

In Figure 7, we represent the height of the intima after 40 iterations for the inflammatory process in the intima. In Figure 6, we represent the deformed domain of the artery after 40 iterations.

\section{CONCLUSiON}

We have built a mathematical model of atheromatous plaque emergence which allows the lesion to grow in particular area in relation with hemodynamics of the blood flow.

Our model is a first step which should be improved in further work in the following direction: we should use a two dimensional model for the growth speed. This could be done by modelling the intima as an abstract fluid with velocity $v(x, t)$ solution of Stokes or more complex visco-elastic equation with a prescribed value for the divergence term. More realistic constitutive laws can also be considered.

\section{REFERENCES}

[1] V. Calvez, A. Ebde, N. Meunier and A. Raoult, Mathematical and numerical modeling of the atherosclerotic plaque formation, in preparation.

[2] N. El Khatib, S. Genieys and V. Volpert, Atherosclerosis initiation modeled as an inflammatory process, Math. Model. Nat. Phenom. (2), 126-141, 2007.

[3] N. El Khatib, S. Genieys, B. Kazmierczak and V. Volpert, Reaction-Diffusion Model of Atherosclerosis Development, to appear.

[4] P. Budu-Grajdeanu, R.C. Schugart, A. Friedman, C. Valentine, A.K. Agarwal and B.H. Rovin, A mathematical model of venous neointimal hyperplasia formation, Theor Biol Med Model. 2008.

[5] B. Osterud and E. BJorklid, Role of monocytes in atherogenesis, Physiol Rev. 83: 1070-1086, 2003.

[6] R. Ross, Atherosclerosis - an inflammatory disease, Massachussets Medical Society 340: 115-120, 1999.

[7] J.M. TARBell, Mass transport in arteries and the localization of atherosclerosis, Annu. Rev. Biomed. Eng. 5: 79-118, 2003. 